\title{
Advance task preparation reduces task error rate in the cuing task-switching paradigm
}

\author{
NACHSHON MEIRAN and ALEX DAICHMAN \\ Ben-Gurion University of the Negev, Beer-Sheva, Israel
}

\begin{abstract}
Advance preparation reduces RT task-switching cost, which is thought to be evidence of preparatory control in the cuing task-switching paradigm. In the present study, we emphasize errors in relation to response speed. In two experiments, we show that (1) task switching increased the rate at which the currently irrelevant task was erroneously executed ("task errors") and (2) advance task preparation reduced the task error rate to that seen in nonswitch trials. The implications of the results to the hypothesis concerning task-specific preparation are discussed.
\end{abstract}

Consider the following set of instructions, typically used in task-switching experiments:

In this experiment, you are asked to perform two tasks [a description of the tasks and of how they are cued follows]. Please respond as quickly as you can without making errors.

In task-switching experiments, such an emphasis on high accuracy is critical because errors most often seem to reflect executions of the wrong task. Moreover, it is critical that participants execute the correct task in trial $n-1$ in order to ensure the correct classification of trial $n$ (the one being analyzed) as involving or not involving a task switch (see Meiran, 1996, Experiments 2 and 3). Assume, for example, a condition in which the required task in trial $n-1$ was letter classification, whereas the erroneously performed alternative task was color classification. An error in this context would reverse the status of trial $n$. If trial $n$ involved color, for example, its status would be changed from "switch" (letter $\rightarrow$ color) to "nonswitch" (color $\rightarrow$ color). Therefore, a high rate of successful task execution is a prerequisite of obtaining a sufficiently large number of analyzable trials.

The emphasis on a low error rate, coupled with the fact that feedback is usually provided for errors but not for slow responses, probably leads participants to pay attention to accuracy rather than response speed. Given the fact that the tasks themselves are often extremely easy, errors probably result from task switching. Hence, it is reasonable to assume that the most critical aspect of successful task control in such paradigms is correct task choice. In the present study, we examine these assumptions.

This research was supported by a research grant from the Israel Science Foundation to the first author. We thank Rotem Eren-Rabinovitch for proofreading the English. Correspondence concerning this article should be sent to N. Meiran, Department of Behavioral Sciences, BenGurion University of the Negev, Beer-Sheva 84105, Israel (e-mail: nmeiran@bgumail.bgu.ac.il).
In spite of the facts that (1) the task-switching paradigm is usually used to study task-control processes and (2) as we have just argued, the most critical aspect of control in this paradigm is response quality and not response time (RT), nearly all previous works have treated accuracy as secondary to RT. Because of the aforementioned considerations, in the present experiments we concentrated on response quality and treated RT as a secondary measure. We believe that this work is relevant to two major theoretical issues in the field, which are outlined below.

\section{Theoretical Issues}

Research on the task-switching paradigm (Allport, Styles, \& Hsieh, 1994; Jersild, 1927; Rogers \& Monsell, 1995; see Monsell \& Driver, 2000) uncovers a variety of robust effects, but the effect that has probably received the most attention in recent literature is task-switching cost. (It should also be mentioned that, although it might be more accurate and theoretically neutral to use the term switching effect - see Ruthruff, Remington, \& Johnson, 2001we have in this case adhered to the traditional term.) The definition of task-switching cost varies somewhat among studies. Nonetheless, a commonly used definition is the difference in performance between trials involving a task switch (switch trials) and trials involving a task repetition (nonswitch trials), both taken from a block of trials in which task switching takes place (mixed-tasks block). Fagot (1994; see also Los, 1996, 1999) suggested a useful scheme in which, in addition to the above-mentioned contrast (switch vs. nonswitch, or switching cost), there was a mixed-list cost contrast between nonswitch trials and trials taken from a block involving one task only (but see Kray $\&$ Lindenberger, 2000, for a different definition). Switching cost reflects dynamic, trial-to-trial effects, whereas mixed-list cost reflects a more ongoing state of readiness for multiple tasks (see, e.g., Braver, Reynolds, \& Donaldson, 2003).

In the short history of task-switching research, a pendulum swings between two seemingly opposing positions regarding the interpretation of task-switching cost. One 
position is that task-switching cost reflects the duration of online control processes. The opposing view is that the cost reflects priming and other processes that do not directly reflect online control processing. Two specific priming hypotheses were most influential. The first, regarding task-set inertia, was formulated by Allport et al. (1994). According to this hypothesis, the task set adopted in the preceding trial is subject to inertia, so that the system takes longer to settle on a unique response choice when the next trial begins. A second variant of the priming hypothesis - the stimulus-task binding hypothesis - was suggested by Allport and Wylie (2000), was implemented in a simulation model by Gilbert and Shallice (2002), and received considerable empirical support from Waszak, Hommel, and Allport (2003). The stimulus-task binding hypothesis suggests that the task set becomes associated with the specific stimuli in response to which the task was executed. In later encounters with these stimuli, the wrong task set tends to be retrieved, which results in a performance interference.

Allport et al. (1994), who argued that task-switching cost does not reflect online control processing, based their conclusion mainly on three pieces of evidence. First, the presumable amount of required task preparation did not affect the size of the task-switching cost in the predicted direction (in their study, switching cost and mixed-list cost were not separated). For example, switching two task aspects (e.g., the relevant stimulus dimension and the response criteria) produced a cost as large as that involved in switching only one task aspect. Second, it was the difficulty of the task in trial $n-1$ rather than the difficulty of that in trial $n$ that affected switching cost in trial $n$. Both of these pieces of evidence have been contested in subsequent articles. Specifically, Hübner, Futterer, and Steinhauser (2001) showed that switching cost increased with the amount of required preparation. Rubinstein, Meyer, and Evans (2001) showed that switching cost increased with the complexity of the task to which the participant switched (see Yeung \& Monsell, 2003b, for a possible resolution of this controversy). The third piece of evidence used by Allport et al. is central to the present article. It concerned the influence of advance task preparation on the size of the task-switching cost. Specifically, in their fifth experiment Allport et al. examined the role of advance task preparation by letting participants perform pairs of trials that involved either a task switch or a task repetition, although in both cases the nature of the trial was known in advance. In addition, they varied the intertrial interval. Presumably, because the task sequence was known in advance, the participants could make use of it and prepare for the task switch. The authors found only a moderate reduction in switching cost in the long intertrial intervals and attributed it to the passive dissipation of the task set adopted in trial $n-1$ rather than to limited advance preparation in trial $n$.

The latter conclusion was contested by Rogers and Monsell (1995) and by Meiran (1996). Rogers and Monsell varied the intertrial interval in a manner similar to that of Allport et al. (1994). Critically, they showed that whether or not advance preparation reduced switching cost depended on the blocking of the preparatory interval. This indicates that the reduction of switching cost was due to strategic factors. Meiran (1996) used the cuing version of the task-switching paradigm (de Jong, 1995; Shaffer, 1965), which is based on a randomly determined task sequence and the presentation of a task cue at the beginning of each trial. This allowed task-set forgetting time, which refers to the interval between the last response and the new target stimulus (related to Allport et al.'s, 1994, hypothesis) to be disentangled from set preparation time. Set preparation time was manipulated by varying the interval between the task cue in trial $n$ and the target in trial $n$ (cue-target interval, or CTI) while keeping set forgetting time (the interval between the $n-1$ response and target stimulus $n$ ) constant. The results indicated a sharp reduction in the task-switching cost due to increasing task preparation time, even when the preparation interval was embedded within a constant set forgetting time.

On the basis of this overadditive interaction between switching and preparation time, reconfiguration theories (see, e.g., Mayr \& Kliegl, 2000; Meiran, 1996, 2000a; Rogers \& Monsell, 1995) assume the existence of switchspecific preparation, or reconfiguration. Nonetheless, these theories typically also assume some form of priming - that is, they assume that the system has a memory for the preceding trial. For example, Rogers and Monsell (1995) used a railroad metaphor for reconfiguration, according to which the role of control is analogous to that of the signalman who heaves the heavy lever that is used to operate the switch between railroad tracks so that the next train will run onto a different line. This metaphor assumes that switching would not be required if the task repeats because the line remains unchanged. In other words, the system "remembers" its state from the preceding trial. Although this assumption concerning system memory was not articulated in terms of priming, it reflects some form of priming nonetheless. Similarly, Meiran (2000a) explicitly assumed that the system retains its reconfiguration from one trial to the next.

Although many alternative hypotheses were ruled out, we would argue that the overadditive interaction between switching and preparation time can still be interpreted in terms of general, nonspecific preparation rather than as reflecting task-switch-specific preparation. This explanation is based on a third variant of the priming theory, which, to our knowledge, has not yet been discussed in the literature (but see Ruge et al., 2005, and Yeung \& Monsell, 2003a, for similar ideas). According to our priming hypothesis, participants who perform in the cuing version of the task-switching paradigm make a task decision on each trial. The reason is that, in this paradigm, the task sequence is not known to the participants in advance. Therefore, a task decision needs to be made even in nonswitch trials. Because task decision takes place in every trial, its duration or difficulty does not affect the switchversus-nonswitch comparison - that is, it does not affect 
switching cost. Switching cost results, according to our new priming hypothesis, from the fact that the task executed in nonswitch trials has been more recently practiced or primed. According to this hypothesis, the reduction in switching cost due to preparation is not task specific (as reconfiguration theories assume) but general in nature. This nonspecific preparation affects switch trials more strongly than nonswitch trials because the switch condition is less practiced, more fragile, and therefore more sensitive to top-down assistance.

The notion that task decision takes place in every trial is supported by studies using the cuing task-switching paradigm in which variables relevant to task decision were manipulated. These variables typically produce RT effects that are additive with that of task switching, indicating that the locus of the effects is in a process common to switch and nonswitch trials. For example, in three studies it was found that if there is a repetitive task sequence that is unknown to the participants, performance improves relative to performance in conditions with an unpracticed task order. Importantly, this learning effect is statistically the same for switch and nonswitch trials (Gotler, Meiran, \& Tzelgov, 2003; Heuer, Schmidtke, \& Kleinsorge, 2001; Koch, 2001). Similarly, in other studies explicit task expectancies were manipulated and their effects were found to be additive with that of switching (Dreisbach, Haider, \& Kluwe, 2002; Ruthruff et al., 2001; Sohn \& Carlson, 2000). Finally, the number of tasks from which to choose affects RT only when little advance preparation is provided, but the effect is statistically equivalent in switch and nonswitch trials (Meiran, Hommel, Bibi, \& Lev, 2002).

The assumption that nonswitch-specific preparation affects switch trials more than nonswitch trials has some support as well. Specifically, although previous comparisons between general preparation and task-specific preparation have shown the independence of the two (Meiran, Chorev, \& Sapir, 2000), the generality of this conclusion may be questioned. First, these authors examined only two forms of general preparation - target onset expectancy and phasic alertness - yet other forms of nonswitchspecific preparation are conceivable. Second, in Meiran, Chorev, and Sapir's study there was a trend, albeit a nonsignificant one, toward a smaller switching cost in conditions with higher alertness. In a series of follow-up experiments, Meiran and Chorev (2005) have shown this small effect to be significant. The fact that the effect was small could be attributed to the weak manipulation of alertness that results from presenting an uninformative stimulus. Kofman, Meiran, Greenberg, Balas, and Cohen (in press) compared performance of students studying in a highly competitive program. The students were tested either during a relatively relaxed period (in the first part of the semester) or during a relatively stressful period (before final exams). These authors found that the preexam period was characterized by increased sympathetic arousal, measured in terms of indices derived from heart rate variability. In- terestingly, this higher level of arousal was accompanied by much greater speeding than that was associated with alertness, as measured in Meiran and Chorev's study. Accordingly, the effect of the increased sympathetic arousal on reducing switching cost was at least twice as large as the effect observed by Meiran and Chorev. Finally, Dreisbach et al. (2002) also argued for differential effects of nonspecific preparation on switch trials, but for a different reason. According to these authors, participants maintain expectancy for task repetitions. Therefore, task repetitions do not enjoy the additional beneficial effects of general readiness, whereas switch trials do.

Note that our new priming hypothesis acknowledges the role of top-down influences and preparation effects but denies the task-switch specificity of these effects, in contrast with reconfiguration theories. This new priming hypothesis would predict that switch trials would be associated with generally poor performance, seen in an increased error rate and lengthened RT. In contrast, the task-set inertial hypothesis of Allport et al. (1994), the stimulus-task binding hypothesis of Allport and Wylie (2000) and Waszak et al. (2003), and several reconfiguration theories (see, e.g., Rogers \& Monsell, 1995; see Mayr \& Kliegl, 2000; Meiran, 2000a) would predict that switching should result in a tendency to repeat the task from the previous trial.

Reconfiguration theories further emphasize task-specific preparation, which counteracts the perseverative tendency to reapply a task set (see especially Yeung \& Monsell, 2003a). In order to support such reconfiguration theories, it would be desirable to observe, directly, whether preparation affects perseveration tendencies, rather than to infer this from RTs and general error rates. As we explained above, general error rates and RTs do not help distinguish between our new priming hypothesis and reconfiguration theories. What differentiates them from each other is the effect of preparation on specific errors. Observation of perseverative tendencies requires the examination of error types, especially those that result from the execution of the wrong task.

\section{Goals and Empirical Focus}

According to reconfiguration theories, advance preparation plays a role in overcoming or counteracting perseverative tendencies such as the tendency to perform the wrong task because the system retains its previous configuration. In contrast, our new priming hypothesis predicts that neither task switching nor readiness time would affect perseveration rates. The critical piece of evidence therefore concerns these perseverations, or task errors. An example of a task error in this paradigm (see Figure 1) is executing the right-left task instead of the required updown task. Note that the emphasis is not on long-term habits, as it is in the Stroop effect (Stroop, 1935) and the Simon effect (Simon \& Small, 1969), for example, but on recently primed tendencies (see especially Hommel, 2000; Marble \& Proctor, 2000). 


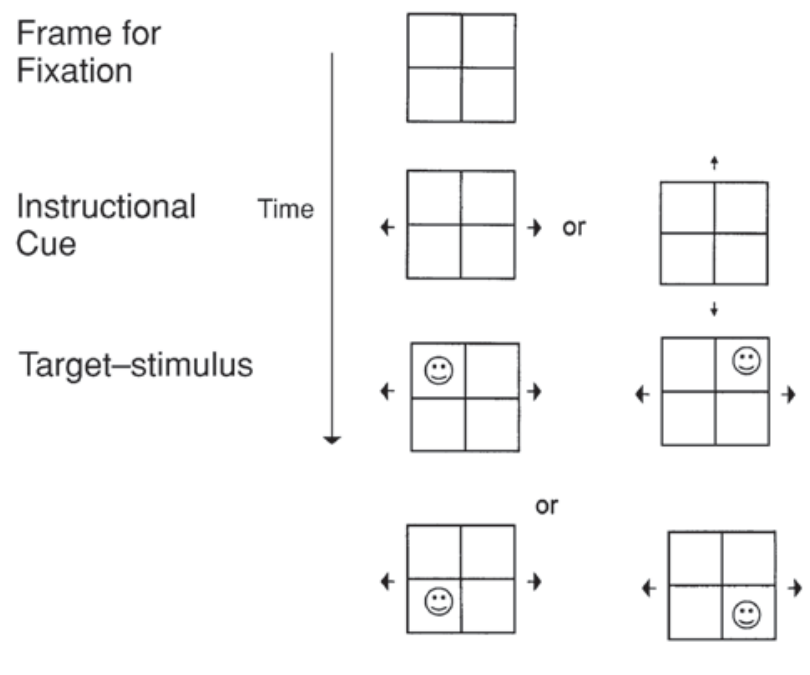

Univalent Response Setup
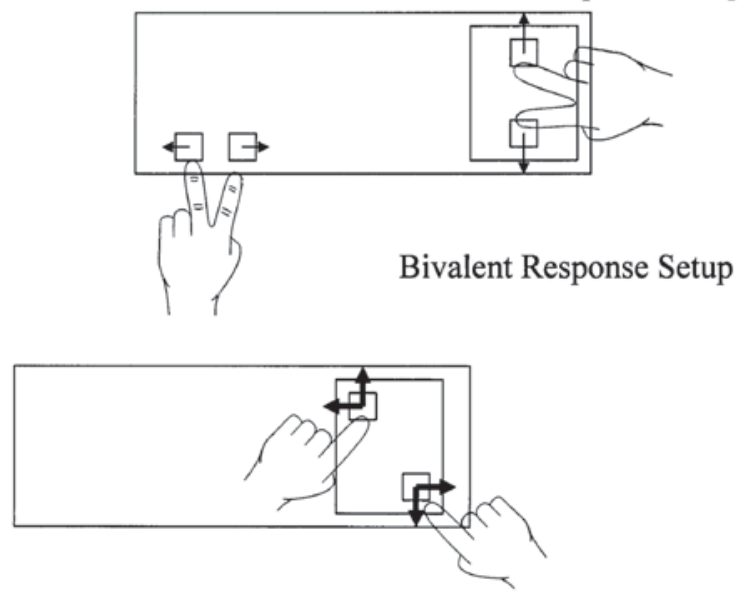

Figure 1. The spatial cuing task-switching paradigm with both of the response setups (univalent and bivalent).

\section{The Present Study}

In two previous studies (Meiran, Gotler, \& Perlman, 2001; Meiran, Levine, Meiran, \& Henik, 2000), we used univalent response setups (see Figure 1) in which each task was associated with a separate set of responses, making it possible to determine the nature of the errors being committed. The results indicated that task switching led to an increase of the rate of correct applications of the wrong task rule. Although these results support our claims, the relevant analyses were always secondary to the main RT analyses because of the very low error rate in these experiments, reflecting the emphasis on accurate responses. This error rate was so low that it forced collapsing over CTI in Meiran, Levine, et al.'s study of schizophrenia patients. Because of this fact, only the effect of task switching on task errors was demonstrated. In the study by Meiran et al. (2001) on the normally aged, there was a sufficient number of errors to demonstrate that CTI prolongation reduced task error rate.
In the present study, we strongly encouraged the young, healthy adults who participated to react quickly in order to commit a relatively high number of errors. We were not interested in error rate per se. Instead, we concentrated on the types of errors the participants committed. Specifically, we employed the following model as our heuristic in analyzing error types (see Figure 2). It was assumed that processing in the task-switching paradigm involves two contingent choices (in the sense discussed by Biederman, 1972). The first choice involves the task. Fagot (1994) called a similar process "task decision," and Rubinstein et al. (2001) as well as Sohn and Anderson (2001) called it "goal setting." In the present work, we could not distinguish between goal setting and the consequent reconfiguration of the system according to the requirements of this task (but see the General Discussion). The choice of a task is based, to a large extent, on the information provided by the task cue. The second choice is between the two possible responses within the chosen task and is based on the information provided by the target stimulus. Because the model involves contingent information processing, preparation for a particular task precedes response selection both logically and temporally. This assumption accords with the majority of contemporary theories of task switching (e.g., Mayr \& Kliegl, 2000; Meiran, 2000a, 2000b; Meiran, Chorev, \& Sapir, 2000; Rogers \& Monsell, 1995; Rubinstein et al., 2001).

Two experiments are reported. Experiment 1 involved a univalent response setup (Figure 1) in which the responses of the two tasks were separated. Experiment 2 involved both a univalent response setup and a bivalent setup (Figure 1) in which the same responses were used in both tasks. These setups were used in separate experimental sessions. The use of a bivalent response setup was essential for showing that participants prepare for a task rather than merely preparing a responding hand or finger.

The major change relative to previous studies from our lab (i.e., Meiran et al., 2001, and Meiran, Levine, et al. 2000 ), other than the speeded instructions, was how we defined task errors. In the previous works, task error was defined as the correct execution of the wrong task. This definition is problematic from the present perspective because it combines the task-selection level with the responseselection level. Specifically, because of our greater interest in task choice, errors in which the correct task was chosen but the correct response within that task was not should be qualified as correct task choices. For this reason, the definition of task errors was changed to reflect the execution of the wrong task regardless of whether this was an accurate or an inaccurate application of the task rule. An example will help clarify this distinction. Assume a trial requiring a right-left decision and a target stimulus in the upper left corner (Figure 2). The correct response in this trial should be "left" because the instructed task is right-left. However, participants may erroneously respond "right," "up," or "down." In our previous articles, we regarded only an "up" response as a task error because "up" would have been a correct response had the required 


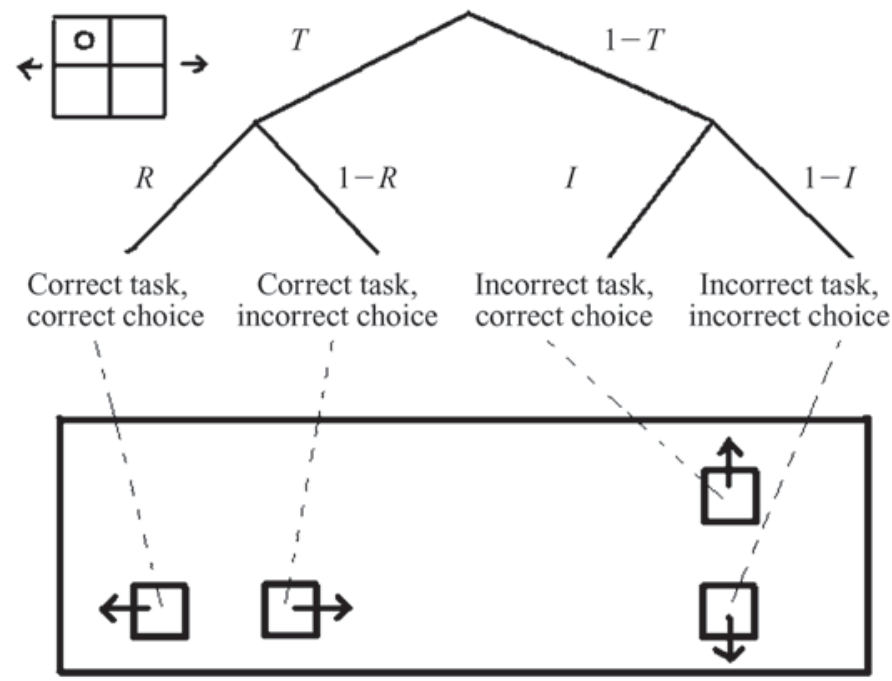

Figure 2. Definition of the primary parameters for the univalent response setup.

task been up-down rather than right-left. However, in the present article, the responses "up" and "down" qualified as task errors. Accordingly, our primary dependent variable $(T)$ was the rate at which the correct task was executed. In this example, it would have been $\left(N_{\text {LEFT }}+\right.$ $\left.N_{\text {RIGHT }}\right) /\left(N_{\text {LEFT }}+N_{\text {RIGHT }}+N_{\text {UP }}+N_{\text {DowN }}\right)$, where $N$ represents the number of trials involving a given response. The rate at which the wrong task was executed (task error rate) is therefore $1-T$. Note that in the model we used, choosing one task came at the expense of choosing the other task. In order to capture the difference between responses within a given task rule (e.g., between "up" and "down"), we specified two additional parameters. One was the conditional probability of correctly executing the relevant task $(R)$, had this task been selected. In the example discussed here (and presented in Figure 2), $R$ equaled $N_{\mathrm{LEFT}} /\left(\mathrm{N}_{\mathrm{LEFT}}+\right.$ $\mathrm{N}_{\text {RIGHT }}$ ). The other variable was the conditional probability of correctly executing the irrelevant task $(I)$, had that task been erroneously selected. In the present example, $I$ was computed as $N_{\text {UP }} /\left(N_{\text {UP }}+N_{\text {DowN }}\right)$. Accordingly, the task error rate, as defined in our previous studies, equals $(1-T) \times I$.

Once the dependent variables are clearly defined, we can derive predictions from the two main hypotheses that we initially contrasted. According to the two dominant priming theories (Allport et al., 1994; Allport \& Wylie, 2000) as well as reconfiguration theories (see, e.g., Rogers \& Monsell, 1995), task switching should result in an increased rate of task errors - that is, lower $T$. Reconfiguration theories also emphasize the increase in $T$ in switch trials as a result of advance preparation. In contrast, our new priming hypothesis predicts that task switching would not affect $T$, because task decisions take place in every trial. The new hypothesis may be consistent with an effect of preparation on $T$, because the task decision pre- sumably begins as soon as the task cue is presented and, in conditions with short CTI, the presentation of the target may interfere with task decision. The hypothesis makes an interesting prediction concerning $R$ and $I$. Because the hypothesis assumes that the more recently practiced task is executed with greater efficiency, $R$ should be greater in nonswitch trials, but $I$ should be greater in switch trials, in which executing the wrong task means executing a task that was more recently practiced.

\section{EXPERIMENT 1}

\section{Method}

Participants. Twelve 1st-year Ben-Gurion University students took part in this experiment in return for course credit. All the participants reported that they had normal or corrected-to-normal vision and did not suffer from any learning disabilities.

Stimuli and Apparatus. All testing was performed using an IBM clone controlled by software written in MEL (Schneider, 1988). Responses were collected with a standard keyboard, and the claimed accuracy in RT recording is to the nearest $1 \mathrm{msec}$. The stimuli were drawn in white on a black background using the graphic symbols in the extended ASCII code and included a $2 \times 2$ grid, presented at the screen center, that subtended a visual angle of approximately $3.4^{\circ} \times$ $2.9^{\circ}$ (values were calculated assuming an observation distance of $60 \mathrm{~cm}$ ). The target stimulus was the smiling face character (ASCII Code 1 ), which subtended approximately $0.3^{\circ} \times 0.5^{\circ}$. The arrowheads (ASCII Codes 16, 17, 30, and 31) subtended approximately $0.3^{\circ} \times 0.3^{\circ}$ and were positioned $0.7^{\circ}$ from the end of the grid (visual angles were computed assuming a $60-\mathrm{cm}$ viewing distance).

Procedure. The experiment consisted of a single 50-min session. It began with a warm-up block ( 15 trials) followed by a short block of 40 trials that was used to calculate the initial mean RT, which was subsequently used to prompt participants to respond faster. The main experiment consisted of four blocks of 128 trials. In these blocks, every response slower than the mean $+30 \mathrm{msec}$ was followed by a warning of "RESPOND FASTER!" presented on a red background. The constant response-cue interval was $1,100 \mathrm{msec}$, and the cue-target interval varied randomly $(100,300,500$, or $2,500 \mathrm{msec})$. 


\section{Results}

As a rule, we do not discuss effects that are qualified by higher order interactions unless the specific effect is of particular relevance. Alpha level was .05 in all the comparisons.

Standard analyses. The present standard analysis is not the most central to this study, but it is presented at the beginning of this section in order to show that encouraging speed did not dramatically alter the standard pattern of $\mathrm{RT}$ results. The mean RT and proportion of errors (PE) according to CTI and task switch are presented in Figure 3.

In the present analysis, we excluded four observations with RTs greater than $3 \mathrm{sec}$ (.006 of all correct RTs). Interestingly, despite the instructions to speed up responses, the usual pattern of RT results was obtained. There were significant main effects for CTI $\left[F(3,33)=60.14, M S_{\mathrm{e}}=\right.$ $1,891.26]$ and task switch $\left[F(1,11)=14.40, M S_{\mathrm{e}}=\right.$ 1,980.51]. Critically, the two variables interacted significantly $\left[F(3,33)=29.51, M S_{\mathrm{e}}=374.11\right]$, showing a reduction in switching cost in the long CTIs. In a parallel analysis of PE, there were significant main effects for CTI $\left[F(3,33)=22.80, M S_{\mathrm{e}}=0.002\right]$ and task switch $\left[F(1,11)=21.00, M S_{\mathrm{e}}=0.0046\right]$, as well as a significant interaction between the two variables $[F(3,33)=18.47$, $\left.M S_{\mathrm{e}}=0.0012\right]$. Note that in the longest CTI, the RT and PE task-switching effects were numerically slightly reversed, showing faster and more accurate switch rather than nonswitch responses. These results are very similar to Meiran's (2000b; see also Brass et al., 2003), showing
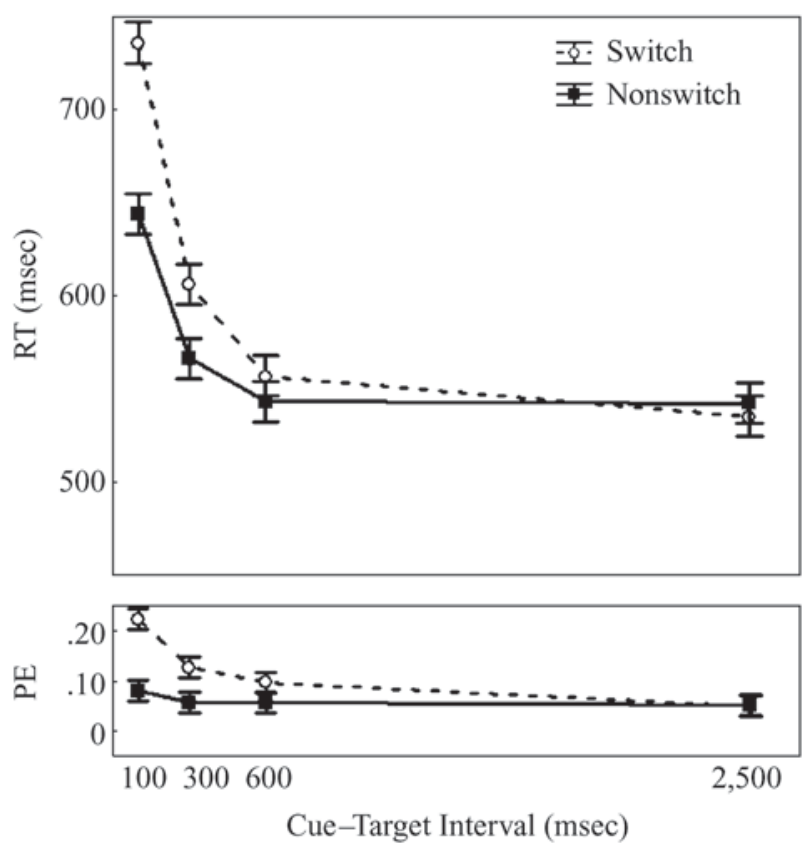

Figure 3. Mean response time (RT, in milliseconds) and proportion of errors (PE) as a function of cue-target interval and task switch in Experiment 1. The .95 confidence intervals are based on the $M S_{\mathrm{e}}$ of the triple interaction. no "residual" switching cost in this paradigm when univalent response setups are being used.

Core analyses. We computed the three parameters defined in Figure 2 as described above. The mean parameter values according to CTI and task switch are given in Figure 4 . The rate of executions of the wrong task, or perseverative errors, is represented by $1-T$. The database had 12 (participant) $\times 4(\mathrm{CTI}) \times 2$ (switch) values, for a total of 96 . Only 22 of these values (23\%) indicated zero task errors. Moreover, all the participants showed task errors in some conditions, and all of them showed task errors in the condition most likely to result in such errors: switch coupled with shortest CTI.

The $T$ and $R$ values, computed separately for each participant, were submitted to two-way ANOVAs according to CTI and task switch. For $T$, there were significant main effects of CTI $\left[F(3,33)=18.73, M S_{\mathrm{e}}=0.001\right]$ and task switch $\left[F(1,11)=19.78, M S_{\mathrm{e}}=0.0017\right]$ as well as a significant interaction between the two variables $[F(3,33)=$ $\left.14.07, M S_{\mathrm{e}}=0.0009\right]$. For $R$, the two main effects were significant [for CTI, $F(3,33)=15.29, M S_{\mathrm{e}}=0.0007$; for task switch, $\left.F(1,11)=15.16, M S_{\mathrm{e}}=0.0016\right]$. The interaction between task switch and CTI was also significant $\left[F(3,33)=7.26, M S_{\mathrm{e}}=0.0005\right]$. Because not all the participants committed task errors in every condition, it was impossible to conduct a similar analysis on $I$. We therefore conducted an analysis on the frequency table collapsed over participants. The analyses indicated that $I$ was significantly affected by switching [continuity corrected $\left.\chi^{2}(1)=7.21\right]$. As our new priming hypothesis predicts, $I$ was greater in switch trials (.89) than in nonswitch trials (.76). However, as can be seen in Figure 4, this was true for CTI $=100$ and $600 \mathrm{msec}$ only. The effect of CTI on $I$ was nonsignificant $\left[\chi^{2}(3)=5.26\right.$ and 6.45 for switch and nonswitch trials, respectively].

\section{Discussion}

We observed that in nonswitch trials the chance of incorrectly executing the wrong task $(1-T)$ was only $.02-.03$. Task switching increased this rate to .13, a value roughly four to six times greater. Most importantly, advance preparation restored the .02 level in $T$. This restoration was gradual and unfolded over time. Because we analyzed proportions, unfolding over time could mean either a gradual change in every trial or a gradual change in the probability that the task would be reconfigured in a given trial, with reconfiguration having an all-or-none quality. The present experiments, however, were not designed to help decide between these two optional interpretations. In this experiment, restoration was found to be nearly complete only when the CTI was sufficiently long $(600 \mathrm{msec})$. The rate at which the $T$ switching cost was affected by CTI was very similar to that at which the RT switching cost was so affected. This observation suggests that the reduction of RT switching cost mirrors advance preparation, although perhaps not as closely as the reduction of $T$ switching cost. One possible explanation for this coupling is that participants slow down under conditions in which 

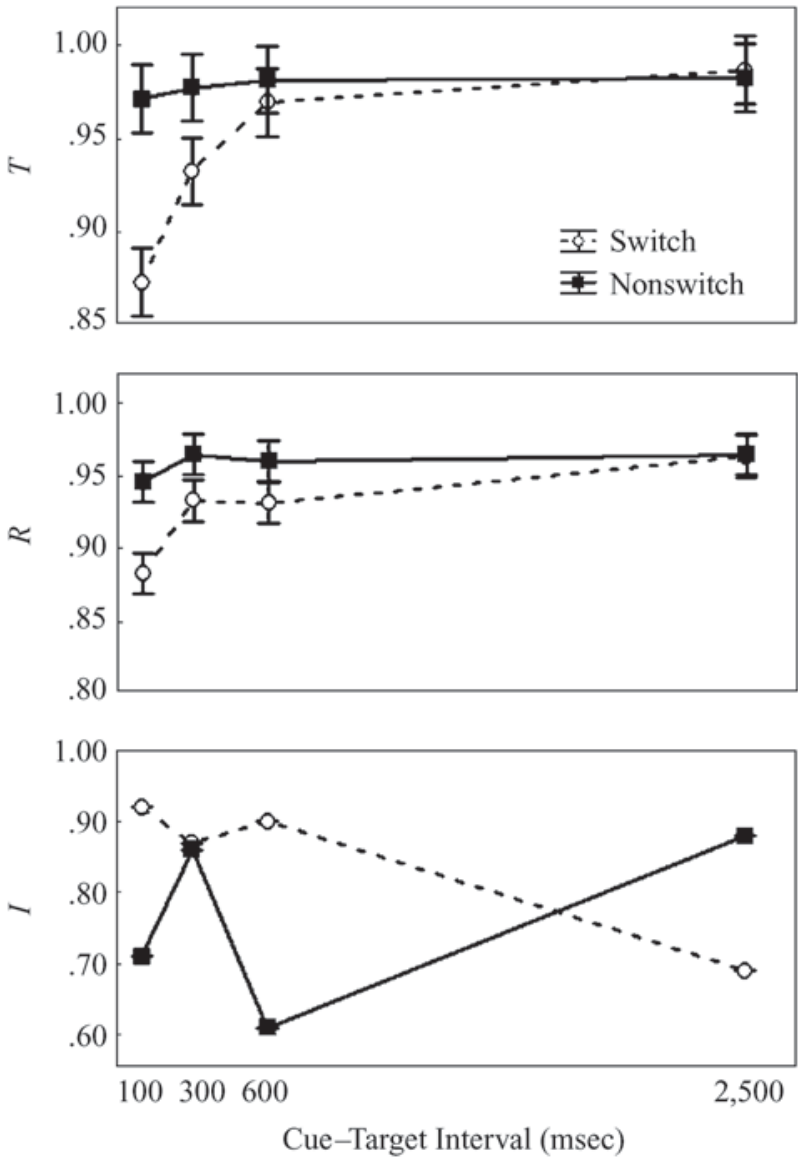

Figure 4. Model parameters as a function of cue-target interval and task switch in Experiment 1. The $\mathbf{. 9 5}$ confidence intervals are based on the $M S_{\mathrm{e}}$ of the two-way interaction.

they are likely to err (see Hillstrom \& Logan, 1997, and Logan, 1994, for discussions of this control strategy). We also observed a similar pattern in the $R$ switching cost, but we will defer a discussion of this aspect until after the entire empirical picture has been presented.

\section{EXPERIMENT 2}

The design of Experiment 1 does not permit us to rule out the possibility that the effects of advance task preparation reflected an effector (i.e., hand or finger) preparation (in the sense discussed by Rosenbaum, 1980, for example). The main goal of Experiment 2 was to study how task switching and advance task preparation affect $T$ estimates when the two tasks share the same overt responses. Such a demonstration would imply that advance task preparation affects the implementation of the task at the cognitive "task rule" level rather than merely at an effector level. This was achieved by using a bivalent response setup in which the same set of two response keys served in both tasks (Figure 1).
The problem with studying bivalent response setups is that it is impossible to directly observe $T$ as in the univalent response setup used in Experiment 1. Therefore, $T$ must be estimated using an explicit processing model. To this end, we employed the processing model presented in Figure 2 but applied it to a bivalent response setup. We capitalized on the fact that half of the trials were task congruent (Figure 5A) and half were task incongruent (Figure 5B; see Sudevan \& Taylor, 1987). Loosely speaking, congruent trials are those in which a correct response could be generated by applying the wrong task rule. In Figure 5, a target in the upper left corner would receive the same correct response regardless of whether the right-left rule or the up-down rule is applied. In contrast, correct responses in incongruent trials depend on applying the correct task rule. Figure 5 tells the more complete story. Specifically, in congruent trials the correct response (the upper left key in Figure 5A) may be generated either by correctly applying the correct task rule or by correctly applying the incorrect task rule (a fact captured by our loose definition). Errors in these trials are generated by an incorrect application of either rule. In incongruent trials, the correct response (the lower right key in Figure 5B) may reflect the correct application of the correct rule or the incorrect application of the incorrect rule. Errors are generated by incorrect application of the right rule or by correct application of the wrong rule. The equations that represent these relations are presented in Table 1 . The present model is a multinomial processing tree (MPT; Batchelder \& Riefer, 1999; Riefer \& Batchelder, 1988; see also Lindsay \& Jacoby, 1994, and the related criticism by Hillstrom \& Logan, 1997, which led us to adopt the MPT).

MPTs are used to estimate the fit of a model to a set of data as well as to estimate the process parameters. The data set consists of the number and proportion of trials falling into each response category. In the present case, there were four response categories for each CTI $\times$ task switch combination. These include correct and incorrect trials in the congruent and the incongruent conditions. The model parameters can be used to predict the proportion of trials falling into each of these response categories. This is done by multiplying the probability values along each path. In some cases, a trial may fall into a given response category for different reasons; each reason is represented as a separate path in the model. For example, a correct congruent response may be generated either by the correct application of the correct rule $(P=T \times R)$ or by the correct application of the incorrect rule $[P=(1-T) \times I]$. Thus, according to the model, the probability of making a correct congruent response is given by the sum of the probabilities above: Expected $P$ (correct $\mid$ congruent $)=T \times$ $R+(1-T) \times I$.

Model estimation is based on comparing the model predictions, as specified above, to the actual numbers in the data set. Specifically, one estimates the likelihood of obtaining the given data given that the model parameters represent population values (see Myung, 2003). To es- 
A

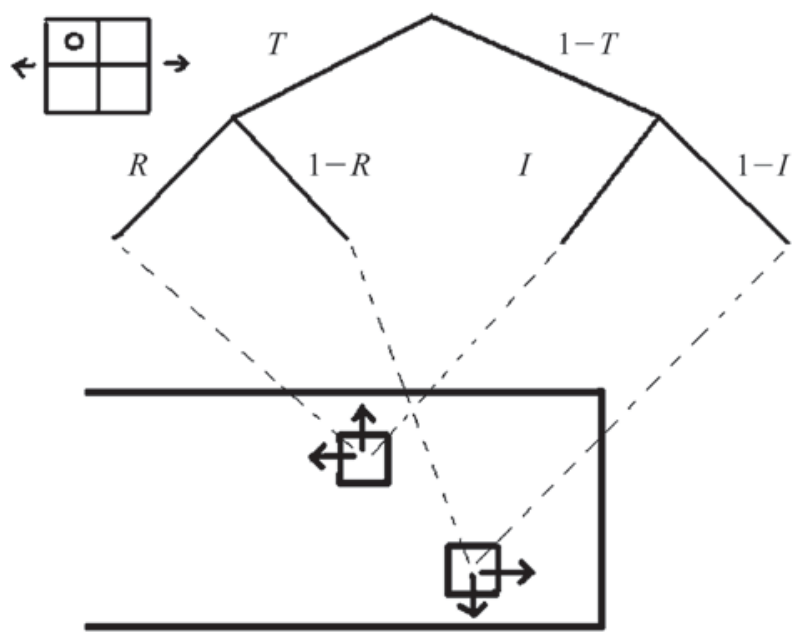

B

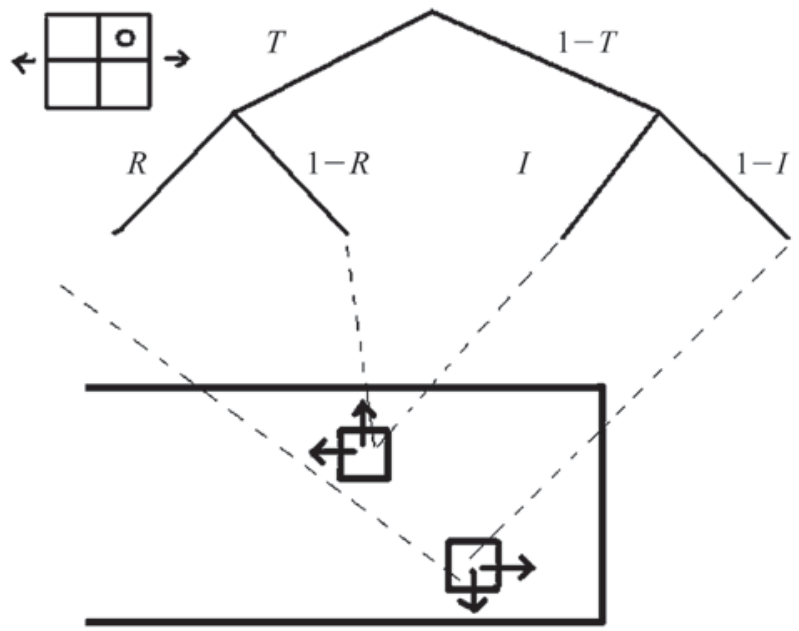

Figure 5. Processing tree model for (5A) the congruent trials and $(5 B)$ the incongruent trials in the bivalent response setup.

timate this likelihood, one needs an explicit probability density function; in this case, the multinomial distribution is used. One then uses search algorithms (we used those in Excel Solver), which are intended to maximize the aforementioned likelihood by changing the model parameters. Finally, one derives a model fit statistic, $G^{2}$, which is distributed approximately similarly to $\chi^{2}$. The degrees of freedom for the test are based on the number of independent pieces of data information (or informations) minus the number of estimated parameters. For example, for each condition there are two proportions - $P$ (correct) and $P$ (incorrect) - but they represent one information only because $P($ correct $)=1-P($ incorrect $)$.

This $G^{2}$ statistic tests the significance of the lack of fit of the model. If the lack of fit is significant, the deviation between the model-based expectancies and the data cannot be attributed to sample fluctuations, meaning that the model is inaccurate. Models can also be compared statistically, yielding a $G^{2}$ statistic for the difference in fit between the models. This, however, can be done only with nested models, in which the parameters of one model consist of a subset of the parameters of the other. In effect, the model comparison procedure is used to test whether or not the addition of a group of new parameters improves the fit significantly.

Another important issue to consider is that the analysis presented in Figure 5 assumes that the congruency variable applies only at the task-rule level. However, one could argue that the analysis misses the fact that, in the spatial task-switching paradigm, this variable may reflect a Simon-like effect (Simon \& Small, 1969). Specifically, the classic Simon effect refers to the fact that, in a nonspatial task, the compatibility of the response position with the irrelevant spatial position of the target stimulus affects performance, showing faster response for spatially compatible targets. In the present paradigm, if the required task is, for example, up-down, the target position on the irrelevant horizontal dimension may affect performance (see especially Nicoletti \& Umiltà, 1984, 1985, and, more recently, Hommel, 1996; Vu \& Proctor, 2001, 2002). Nonetheless, the present approach is defensible on the basis of the results of a recent paper by Meiran (2005), who compared two response setups. One was the standard setup presented in Figure 1, in which the position of the response key is compatible with that of the target along the relevant dimension (e.g., an upper key indicating UP). The other response setup had a reversed mapping (e.g., the upper key indicating DOWN). This mapping reversal manipulation reverses the potential Simon-like effect but keeps the potential rule-congruency effect intact. The RT results displayed nearly perfect additivity of mapping reversal and rule congruency, indicating no Simon-like influence on the rule congruency effect.

It should be pointed out that even if we find the predicted trend in the bivalent response setup, the results of Experiment 1 do not necessarily reflect task prepara-

Table 1

Equations Derived From the Model Presented in Figure 5

\begin{tabular}{cll}
\hline Condition & \multicolumn{1}{c}{ Definition } & \multicolumn{1}{c}{ Equation } \\
\hline E1 & $P$ (correct|congruent) & $T \times R+(1-T) \times I$ \\
E2 & $P$ (incorrect|congruent $)$ & $T \times(1-R)+(1-T) \times(1-I)$ \\
$F_{1}$ & $P($ correct|incongruent $)$ & $T \times R+(1-T) \times(1-I)$ \\
$F_{2}$ & $P($ incorrect|incongruent $)$ & $T \times(1-R)+(1-T) \times I$ \\
\hline
\end{tabular}


Table 2

Mean Response Times (RTs, in Milliseconds) and Proportions of Errors (PEs) According to Condition in Experiment 2

\begin{tabular}{|c|c|c|c|c|c|c|c|c|c|c|c|c|c|}
\hline \multirow[b]{4}{*}{ Condition } & \multirow[b]{4}{*}{ CTI (msec) } & \multicolumn{12}{|c|}{ Response Setup } \\
\hline & & \multicolumn{6}{|c|}{ Univalent } & \multicolumn{6}{|c|}{ Bivalent } \\
\hline & & \multicolumn{2}{|c|}{ Switch } & \multicolumn{2}{|c|}{ Nonswitch } & \multicolumn{2}{|c|}{ Cost } & \multicolumn{2}{|c|}{ Switch } & \multicolumn{2}{|c|}{ Nonswitch } & \multicolumn{2}{|c|}{ Cost } \\
\hline & & RT & $\mathrm{PE}$ & RT & $\mathrm{PE}$ & RT & $\mathrm{PE}$ & RT & $\mathrm{PE}$ & RT & $\mathrm{PE}$ & RT & $\mathrm{PE}$ \\
\hline \multirow{3}{*}{ Unspeeded } & 100 & 656 & .10 & 577 & .04 & 79 & .06 & 707 & .07 & 604 & .01 & 103 & .06 \\
\hline & 600 & 517 & .01 & 523 & .02 & -6 & -.01 & 544 & .03 & 527 & .02 & 17 & .01 \\
\hline & 2,500 & 503 & .04 & 500 & .01 & 3 & .03 & 552 & .04 & 533 & .02 & 19 & .02 \\
\hline \multirow[t]{3}{*}{ Speeded } & 100 & 609 & .13 & 540 & .03 & 69 & .10 & 587 & .09 & 531 & .04 & 56 & .05 \\
\hline & 600 & 470 & .04 & 471 & .02 & -1 & .02 & 492 & .06 & 481 & .04 & 11 & .02 \\
\hline & 2,500 & 474 & .02 & 471 & .03 & 3 & -.01 & 496 & .06 & 481 & .04 & 15 & .02 \\
\hline
\end{tabular}

tion as opposed to hand preparation. It is conceivable that the participants in Experiment 1 used hand preparation whereas those in the present experiment used rule preparation. In order to address the latter possibility, the participants performed in both a univalent and a bivalent response setup, which made it possible to compare the results of the two setups within the same group of participants. A similar pattern of results would suggest (but not prove) that the same type of preparation was used in both setups. In addition, in Experiment 2 we improved the reliability of the results by increasing the number of participants and the number of trials per condition. This was achieved by using three CTIs instead of four (so that there are more observations in each CTI condition) and by running participants in four sessions, with two sessions per condition (univalent vs. bivalent responses) rather than one session, as was the case in Experiment 1.

\section{METHOD}

\section{Participants}

Twenty undergraduate students took part in this experiment; they had attributes similar to those who participated in Experiment 1. The participants were assigned to four counterbalancing schemes according to their order of entry into the experiment. The schemes were based on the combination of two testing orders (bivalentunivalent-univalent-bivalent vs. univalent-bivalent-bivalentunivalent) and, in the bivalent condition only, two response key combinations (upper left and lower right vs. upper right and lower left).

\section{Stimuli and Procedure}

These were the same as in Experiment 1 except that there were four identical sessions instead of just one, and three CTIs (100, 600, and 2,500 $\mathrm{msec}$ ) instead of four.

\section{RESULTS}

\section{Standard Analyses}

Although in Experiment 1 we encouraged the participants to speed up their responses at the expense of accuracy, the design of that experiment prohibited us from concluding (1) whether or not the manipulation had an effect and (2) whether or not it modulated the effects of interest. To this end, we capitalized on the multisession structure of the present experiment and used the second block (in which speeding messages were not yet displayed) of Sessions 2-4. This block was excluded from Session 1 in order to roughly equate the speeded (S) and unspeeded (US) conditions for practice effects. Viewed from this perspective, the order of conditions being analyzed was S1-US2-S2-US3-S3-US4-S4 (the digits 1-4 represent the sessions). The results were analyzed in an ANOVA with the independent variables of condition (speeded vs. unspeeded), response valence (univalent vs. bivalent), CTI (100 vs. 600 vs. 2,500 msec), and task switch (switch vs. nonswitch). Mean RT and PE according to condition are presented in Table 2.

Response time. Not surprisingly, there was a main effect of condition, with speeded responses being $53 \mathrm{msec}$ faster $(509 \mathrm{msec})$ than unspeeded responses $[562 \mathrm{msec}$; $\left.F(1,19)=17.79, M S_{\mathrm{e}}=19,164.03\right]$. The usual main effects, including those of CTI $\left[F(2,38)=85.23, M S_{\mathrm{e}}=\right.$ $6,177.51]$ and task switch $\left[F(1,19)=23.89, M S_{\mathrm{e}}=\right.$ $4,720.44]$, were significant, and so was their interaction $\left[F(2,38)=28.93, M S_{\mathrm{e}}=2,231.57\right]$. This interaction was not significantly affected by condition, as can be seen in the nonsignificant triple interaction of condition, CTI, and task switch $\left[F(2,38)=1.82, M S_{\mathrm{e}}=1,406.72, p>.15\right]$. The CTI $\times$ task switch interaction was also unaffected by response valence, as is indicated by a nonsignificant triple interaction of the three variables ( $F<1$; see Figure 6$)$. Finally, the combination of response valence and condition also did not modulate the task switch $\times$ CTI interaction, as can be seen in a nonsignificant quadruple interaction of the four variables $(F<1)$.

Response valence and task switch produced a marginally significant interaction in the predicted direction $\left[F(1,19)=4.19, M S_{\mathrm{e}}=814.36, p=.055\right]$. That is, switching cost was larger when the response setup was bivalent (563 vs. $526 \mathrm{msec}$, or $37 \mathrm{msec}$ ) than when it was univalent (538 vs. $513 \mathrm{msec}$, or $25 \mathrm{msec}$; see Figure 6A). This is the same result reported by Meiran (2000b; see Brass et al., 2003).

Condition produced a triple interaction with response valence and CTI $\left[F(2,38)=4.15, M S_{\mathrm{e}}=1,831.31\right.$; see Figure 7], which qualified as a lower order interaction between condition and $\mathrm{CTI}\left[F(2,38)=4.82, M S_{\mathrm{e}}=1,684.85\right]$. In addition, there was a marginal interaction between condition and task switch $\left[F(1,19)=4.19, M S_{\mathrm{e}}=814.36, p=\right.$ 

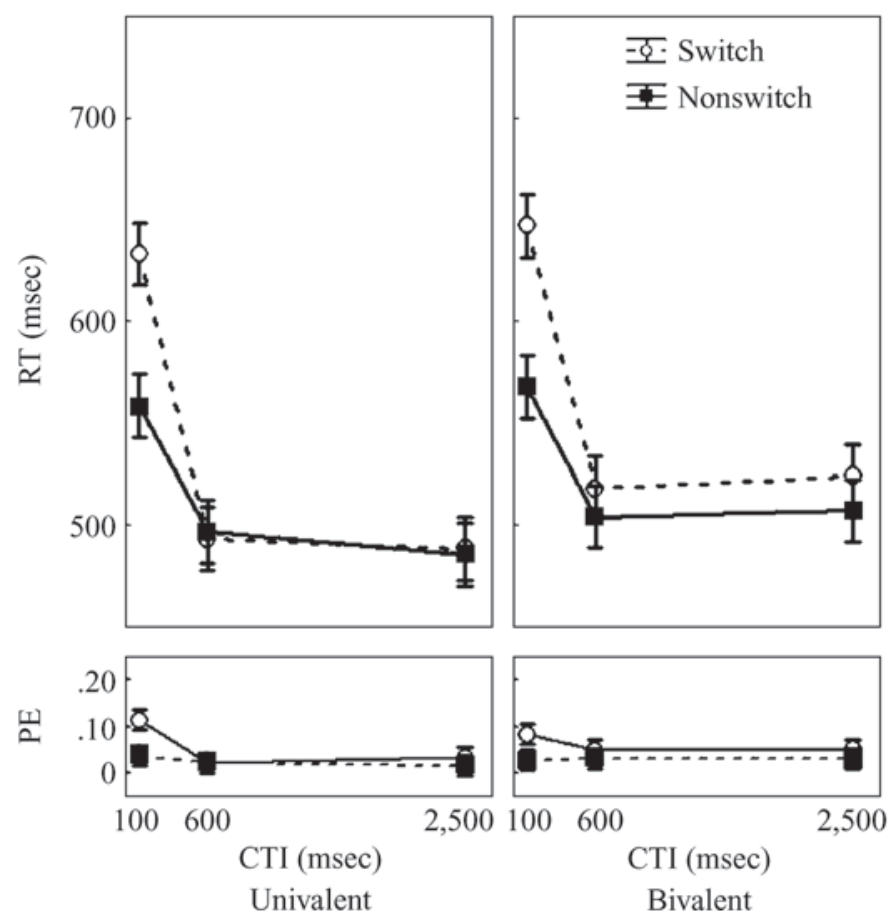

Figure 6. Mean response time (RT) and proportion of errors (PE) as a function of cue-target interval (CTI) and task switch in Experiment 2. The .95 confidence intervals are based on the $M S_{\mathrm{e}}$ of the nonsignificant triple interaction.

.055]. The marginal interaction reflects smaller switching cost under speeded conditions $(25 \mathrm{msec})$ than under unspeeded conditions $(36 \mathrm{msec})$. This result is similar to that of de Jong (2000), who observed smaller switching costs among the relatively fast responses. We are unsure, however, if the latter interaction is real because, aside from being marginally significant, it was also accompanied by a numerical (but nonsignificant, $F<1$ ) trend toward a speed-accuracy trade-off. Specifically, the speeded condition produced a larger switching cost in PE (.04) in comparison with the unspeeded condition (.03).

Proportion of errors. First, PE rate was significantly higher in speeded conditions (.05) than in unspeeded conditions $\left[.03 ; F(1,19)=11.12, M S_{\mathrm{e}}=0.0193\right]$. Coupled with the RT effect, the results indicate that speeding instructions led to a moderate increase in error rate coupled with faster responses. Additional significant main effects included those of CTI $\left[F(2,38)=11.00, M S_{\mathrm{e}}=0.0055\right]$ and task switch $\left[F(1,19)=40.82, M S_{\mathrm{e}}=0.0028\right]$, respectively. In addition, there were two significant two-way interactions. One, which tended in the same direction as the RT effect, was between CTI and task switch $[F(2,38)=$ $\left.11.22, M S_{\mathrm{e}}=0.0033\right]$; the other was between response valence and CTI $\left[F(2,38)=4.77, M S_{\mathrm{e}}=0.0040\right]$. The univalent response setup produced a PE that was .02 greater than that in the bivalent setup. However, this trend was reversed for the two long CTIs (an effect of .02).

\section{Core Analyses}

Univalent responses. For $T$, there were significant main effects of CTI $\left[F(2,38)=28.75, M S_{\mathrm{e}}=0.0008\right]$ and task switch $\left[F(1,19)=47.24, M S_{\mathrm{e}}=0.0003\right]$. This was also true for $R$ [for CTI, $F(2,38)=13.84, M S_{\mathrm{e}}=$ 0.0003 ; for task switch, $F(1,19)=20.75, M S_{\mathrm{e}}=0.0003$. Critically, the interaction between task switch and CTI was significant for $T\left[F(2,38)=34.10, M S_{\mathrm{e}}=0.0004\right]$ and for $R\left[F(2,38)=17.39, M S_{\mathrm{e}}=0.0002\right]$. As in Experiment $1, I$ was analyzed on the aggregated data. The switching effect was nonsignificant [continuity-adjusted $\left.\chi^{2}(1)=2.53\right]$. The raw trend indicated that $I$ was greater $(.84)$ in nonswitch trials than in switch trials (.83), but the pattern was noisy. The CTI effect was examined on the data collapsed over switching. This test too was nonsignificant $\left[\chi^{2}(2)=4.31\right]$. In this experiment as well, all the participants showed task errors in some conditions and all of them showed it in the switch-short CTI condition. Of the 120 values in the data set [ $3(\mathrm{CTI}) \times 2$ (task switch) $\times$ 20 (participant)], only 24 had a $T$ of 1 ; that is, they had no task errors (see Figure 8 for descriptive statistics).

Bivalent responses. The results were analyzed using Riefer and Batchelder's (1988) multinomial processing tree analyses. We used the equations in Table 1 and Excel Solver software to maximize the likelihood ratio and to obtain $G^{2}$ tests of the model's lack of fit. The $G^{2}$ is distributed approximately as $\chi^{2}$ with degrees of freedom equal- 

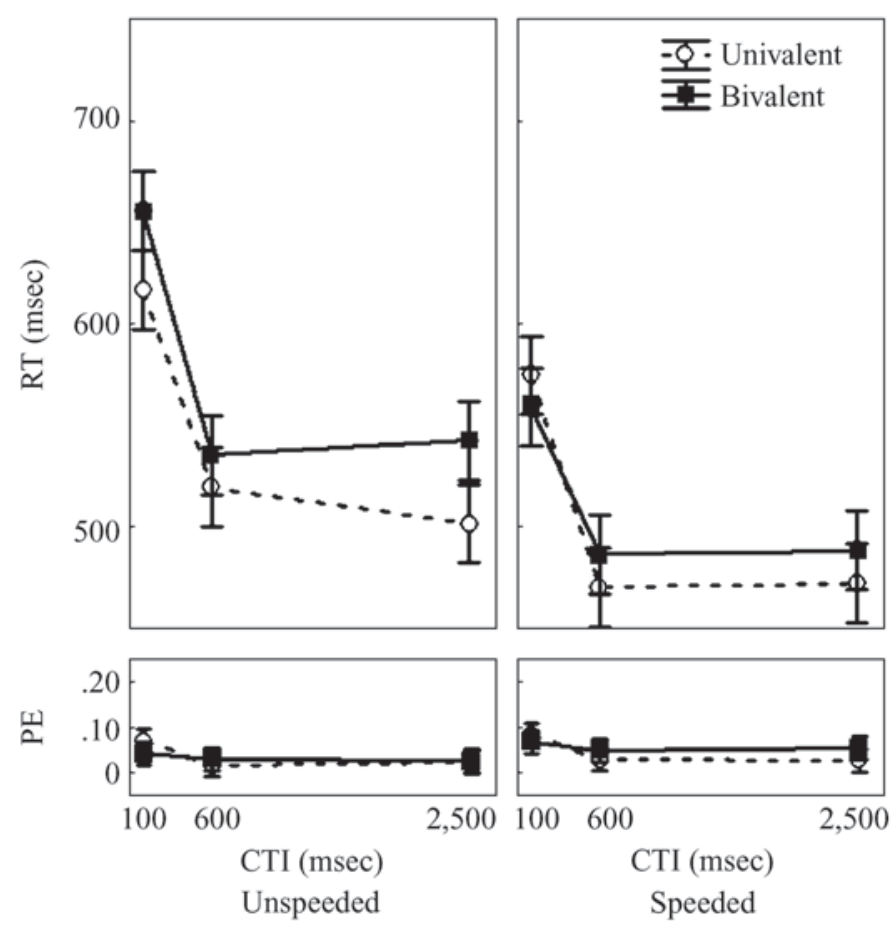

Figure 7. Mean response time (RT) and proportion of errors (PE) as a function of condition (speeded vs. unspeeded), cue-target interval (CTI), and response valence in Experiment 2. The .95 confidence intervals are based on the $M S_{\mathrm{e}}$ of the triple interaction.

ing the number of informations minus the number of free parameters. In the present case, only the proportions of correct trials are considered as informations because the proportions of errors are given by $1-P$ (correct).

The raw data consisted of the response counts in the large table of raw results formed by crossing participant, CTI, switch, congruency, and response correctness (correct vs. incorrect). (In order to make estimation possible, we replaced the 71 zero counts, out of 480 matrix entries, with the negligible value .000001). We first tried our Model 1 (henceforth, MDM-1). MDM-1 included only three free parameters: $T, R$, and $I$. This means that the model assumed that $T, R$, and $I$ were the same for all CTIs and all participants and for both the switch and nonswitch conditions. Unsurprisingly, MDM-1 produced an unsatisfactory fit, with a significant deviation between model and data $\left[G^{2}(237)=1,217.52\right]$. In the second stage, we tried Model 2 (MDM-2), which included $R, I$, and two separate $T$ parameters, one for switch and one for nonswitch. In that respect, MDM-2 was like MDM-1 except that the former loosened the constraint on an equal number of task errors $(1-T)$ in the switch and nonswitch conditions. Although the fit of MDM-2 $\left[G^{2}(236)=1,061.33\right]$ was unsatisfactory, the improvement in fit relative to MDM-1 $\left[G^{2}(1)=\right.$ 156.19] was clearly significant. This comparison between MDM-2 and MDM-1 indicates that switching affected the rate of task errors $(1-T)$ significantly.
Next, we tried Model 3 (MDM-3), which included $R$, $I$, and four different $T$ parameters: one for nonswitch and one for each of the three CTIs within the switch condition. MDM-3 assumes that task switch affects $T$ and that CTI affects $T$ as well, but only in switch trials. This model produced an unsatisfactory fit, with significant deviation between model and data $\left[G^{2}(234)=820.17\right]$. The improvement relative to MDM-2 was significant, however $\left[G^{2}(1)=241.17\right]$. Thus, we can conclude that CTI significantly affected $T$ in switch trials. Because MDM-3 produced an unsatisfactory fit, we tried to improve it by adding three free parameters in Model 4 (MDM-4). These included a specific $R$ value for each CTI in the switch condition and one $R$ value representing all the CTIs in the nonswitch condition. In other words, MDM-4 assumed that task switch and CTI affected $R$ as well as $T$, in contrast to MDM-3, which assumed that CTI and task switch affected only $T$. MDM-4 had a significant lack of fit $\left[G^{2}(234)=815.63\right]$ - that is, in the improved fit relative to MDM-3, $G^{2}(3)=4.54$, which was not significant. In other words, switch and CTI did not significantly affect $R$. We therefore tried MDM-5, which was the same as MDM-3 except that it allowed for individual differences in the parameter values. This individually fitted model produced satisfactory fit in all the participants $\left[G^{2}(6)=\right.$ $0.00-9.72, p>.1$, at the individual level, and $G^{2}(120)=$ 35.87 , n.s., at the group level]. The improvement in 

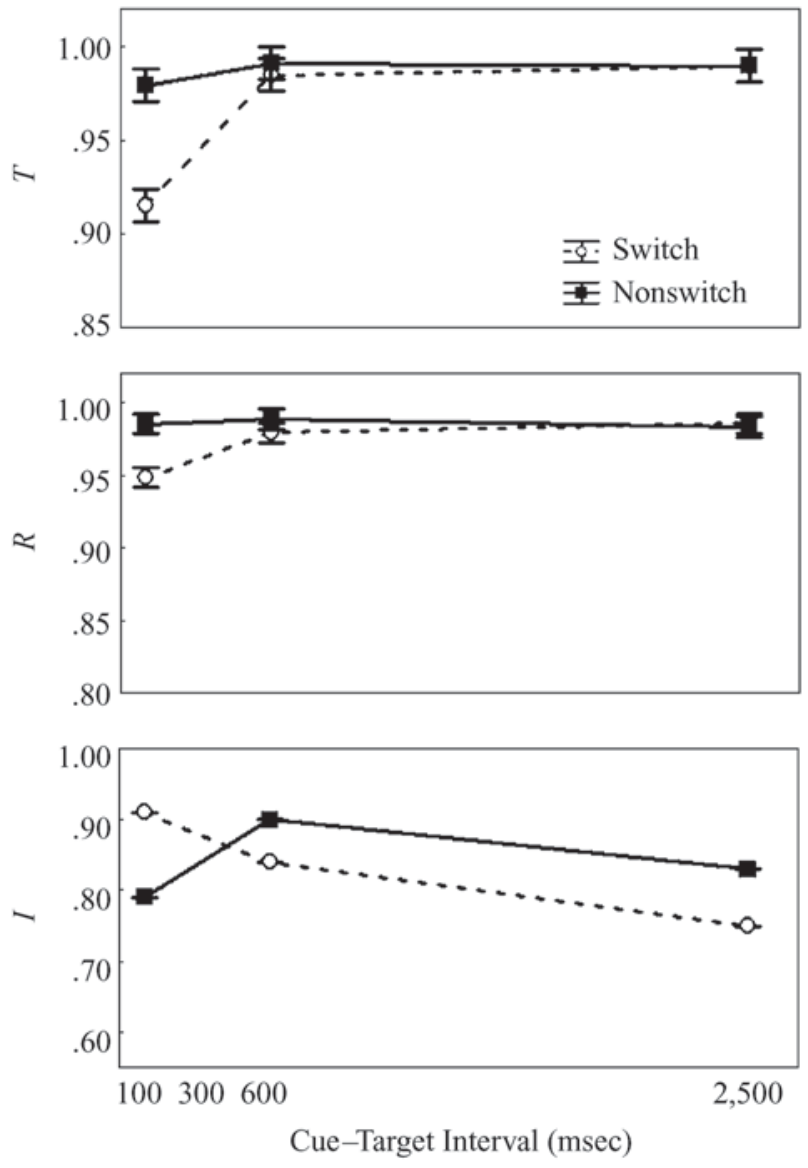

Figure 8. Model parameters as a function of cue-target interval and task switch in the univalent response setup of Experiment 2. The .95 confidence intervals are based on the $M S_{\mathrm{e}}$ of the two-way interaction.

MDM-5 relative to MDM-3 was significant $\left[G^{2}(114)=\right.$ 784.30 at the group level]. This final model comparison indicates that all the participants processed the task in the same way, as far as we could tell, but that there were significant individual differences in the probabilities of correctly choosing the task and correctly executing the relevant and irrelevant rules.

Finally, we examined whether the parameter estimates, based on the aggregated (across-participants) MDM-3, were similar to the mean of the values estimated for each participant (MDM-5). This was true for five of the six parameters in the model, in all of which the aggregated value fell within the .95 confidence interval. (For all but one of the comparisons of the aggregated MDM-3 estimates with the mean individual estimates from MDM-5, $t<$.7.) The only exception was $R$, for which the mean individual parameter was .995 as opposed to .999 in the aggregated model $[t(19)=3.39, S E=0.0015]$. Because this difference was tiny, albeit significant, we present the aggregated parameters of MDM-3. Figure 9 depicts the switch and CTI effects on $T$ in the bivalent (MDM-3) response setups. As can be seen, the $T$ values in the bivalent response setup were very similar to those in the univalent response setup. In both cases, advance preparation eliminated the switch effect on $T$, with switch still producing a slight effect in the intermediate CTI. The only substantial difference between the two sets of values consists of a higher rate of task errors (lower $T$ ) in the bivalent response setup than in the univalent response setup. This result is not surprising, given the fact that the sharing of responses in the bivalent response setup probably contributes to task confusion.

\section{Discussion}

The present results replicate and extend those of Experiment 1 . First, we can conclude that encouraging participants to increase their speed worked in the predicted direction, although the effect was very moderate. Perhaps the participants were already in a mindset of trying to work faster when we measured them under ostensibly unspeeded conditions. Still, the error rate was not very high, showing that they tried to maintain a high level of accuracy - evidence that they still applied cognitive control in the sense discussed above. In addition, the speeded condition produced effects that were generally similar to those in the unspeeded condition. This fact allows us to proceed to our main points.

Most importantly, we showed that in the bivalent response setup the presence of task switching roughly doubled the task error rate in switch trials with short CTIs $(1-T=.19)$ in comparison with the error rate in nonswitch trials (.09). Moreover, advance task preparation reduced the task error rate in the switch condition to about one sixth (.03) of its value given short CTIs. This effect is similar to that obtained in the univalent response setup, in which advance preparation reduced the task error rate in switch trials from .09 to .01 (in comparison with .01-.02 in nonswitch trials). Despite this similarity between the response setups, advance preparation affected only $R$ in the univalent response setup.

It may be added that the fact that MDM-5 fit the data well provides moderate support for its underlying assumption. Although a successful fit does not prove the

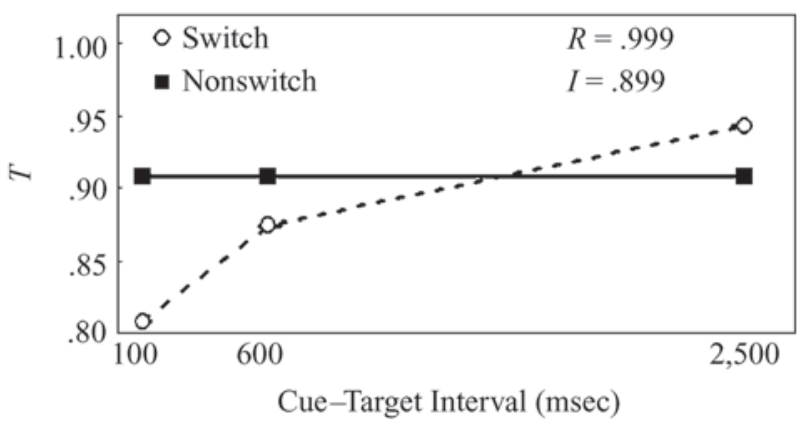

Figure 9. MDM-3 maximum likelihood estimates for the bivalent response setup in Experiment 2. Model comparisons indicated that, in the bivalent setup, $R$ and $I$ were unaffected by switching and cue-target interval. 
model to be correct, it at least supports it and demonstrates its plausibility. Additional support for the model comes from studies such as that of Biederman (1972), who presented participants with stimuli varying in three dimensions: color, size and tilt. In the critical condition, which he called "contingent" and which would today be termed task switching, one of the stimulus dimensions served as a task cue indicating which of the remaining dimensions was relevant. This condition was compared to a lower and an upper baseline condition. In the lower baseline (i.e., filtering) condition, participants were required to judge along two dimensions while ignoring irrelevant variation along a third dimension. This condition is similar to the contingent condition in requiring that only two dimensions be taken into account. The upper baseline (i.e., condensation) condition required judgments based on the combination of all three dimensions. Biederman's results indicated that RT in the contingent condition was between RT in the two baseline conditions (see also Keele \& Rafal, 2000, Experiment 1).

The assumption that participants do not have a flat representation of the task but, rather, a hierarchical representation in which response selection is nested in its respective task (see Kleinsorge \& Heuer, 1999; Zelazo \& Frye, 1997) is also supported by studies showing that participants can learn the task sequence in the cuing taskswitching paradigm (Gotler et al., 2003; Heuer et al., 2001; Koch, 2001) despite the fact that neither the stimuli nor the responses obey a predetermined order.

\section{GENERAL DISCUSSION}

In the present experiments, we emphasized errors as the critical outcome of control processing in speeded task switching. Specifically, we concentrated on $T$, which is the probability of executing the relevant task as opposed to erroneously executing the irrelevant task $(1-T)$. Our novel results indicate that $T(1)$ was affected by task switching, in that it was smaller in switch trials, and (2) increased with advance preparation in switch trials. In addition, we replicated previous findings concerning the increase of RT in switch trials and its reduction in switching cost due to advance task preparation, which was partial with bivalent response setup (indicating residual cost) and complete with univalent response setups (indicating no residual cost). The implications of these findings will be discussed in the remainder of the General Discussion.

\section{Task-Specific Versus Task-Unspecific Preparation}

In the introduction, we suggested a new priming hypothesis that was both plausible and able to account for the interactive effect of switching and preparation on RT. According to this hypothesis, in the cuing paradigm task decision takes place in every trial and therefore does not affect the RT or PE switching cost. The performance difference between switch and nonswitch trials is explained by the fact that switch trials are associated with the per- formance of a less recently practiced task. This hypothesis predicts that task switching would not affect $T$, because task decisions take place in every trial. Switching is more likely to affect $R$ and $I$. The new hypothesis may be consistent with a general and nonswitch-specific effect of preparation on $T$, because the task decision presumably begins as soon as the task cue is presented. Therefore, in conditions with short CTIs, the presentation of the target may interfere with that decision.

The present results do not support these predictions. Moreover, the interesting prediction of the new priming hypothesis concerning reversed effects of task switching on $R$ and $I$ was also not supported by the data. Instead, the results clearly support the predictions of the two dominant priming theories (Allport et al., 1994; Allport \& Wylie, 2000) and several reconfiguration theories (e.g., Rogers \& Monsell, 1995). All these theories predict that task switching should result in an increased rate of task errors and, hence, lower $T$. The results also support a unique prediction of reconfiguration theories concerning an increase in $T$ in switch trials as a result of advance preparation.

\section{How to Interpret $T$ ?}

Various theories in the literature have addressed two forms of task readiness: task decision and reconfiguration - that is, implementing the proper configuration (Fagot, 1994; Rubinstein et al., 2001; Sohn \& Anderson, 2001; Waszak et al., 2003). Our heuristic model, presented in Figures 2 and 5, represents both task decision and task readiness with a single parameter, $T$, and therefore does not seem to be able to discriminate between these processes. However, on the basis of results from other studies, we would argue that $T$ represents actual task readiness and not task decision. This claim is specific to the random cuing version of the task-switching paradigm, in which participants seem to be required to make a task decision in every trial. Therefore, task decision does not contribute to the switching effect. Evidence of this comes from studies in which implicit task expectancy (Gotler et al., 2003; Heuer et al., 2001; Koch, 2001), explicit task expectancy (Dreisbach et al., 2002; Ruthruff et al., 2001; Sohn \& Carlson, 2000), and number of task alternatives (Meiran et al., 2002), among others factors, are manipulated. All these effects were shown to be additive with the effect of task switching on RT (see Rubin \& Meiran, in press, for direct support). Because $T$ was affected by switching whereas task decision was not, $T$ must reflect actual task readiness.

We therefore suggest the following account of the results. The effects on $T$ can be explained by assuming that, first, the system configuration remains in place (at least partly) after task execution. Second, reconfiguration takes place only after task decision has been accomplished. Moreover, the probability of successful task decision increases with CTI (see, e.g., Logan \& Bundesen, 2003, for an elegant mathematical formulation of similar increases in process completion probabilities), which implies, in turn, that the probability of successful reconfiguration 
also increases. This implies that in some trials the target may be processed before reconfiguration. In nonswitch trials, this would result in execution of the correct task. However, in switch trials, such premature processing would result in a task error. Because increasing task preparation time improves the chances for reconfiguration, it also reduces the chances that target information will be processed according to the wrong rule. It is significant that this account makes it clear that task decision without a consequent reconfiguration would not suffice. It seems vital to assume that, for task errors to occur, the system must be configured according to the wrong task rule.

\section{Effects on $R$ and $I$}

We suggest two possible (but post hoc) accounts for the effects on $R$. Briefly, we found that, for $R$, the interaction between switching and preparation was similar to that found for $T$ when a univalent response setup was used. With a bivalent response setup, $R$ was very high and was affected neither by switch nor by preparation. One hypothesis is that the system's configuration improves incrementally (see, e.g., Meiran, 2000a, for a possible mechanism). This means that the system is more optimally tuned to make a correct response selection in nonswitch trials, which explains the effect of switching. Moreover, once the task has been chosen, there is top-down biasing of the response selection process, which improves the functioning of the process (see, e.g., Waszak et al., 2003; Yeung \& Monsell, 2003a, 2003b). This assumption explains why an increase in CTI resulted in an increase in $R$ in switch trials. Finally, all of the above must take place at a very peripheral motor level, because when task preparation could not involve readying an effector (the bivalent response setup), no effects of switching or preparation were observed on $R$.

Another hypothesis is that the task switch was implemented, at least partly, by the operation of the logical rule of inversion, and that this rule operated indiscriminately, affecting the response level. In other words, switching the task resulted in inverting the correctly chosen response. Similar indiscriminate applications of logical operations were described by Duncan $(1977,1978)$. He studied conditions involving multiple stimuli and multiple responses, so that in some cases the stimulus-response (S-R) rule was that of identity ("respond in the same position as the stimulus") and in others the rule required the application of the "opposite" transformation on stimulus location ("respond in the mirror position relative to that of the stimulus"). Duncan found that a relatively frequent error type reflected the application of the incorrect S-R transformation rule. Another example is given in Kleinsorge and Heuer's (1999) theory of task switching, which assumes that when the tasks are arranged in a logical hierarchy, "switch" signals propagate uncontrollably downward in the task hierarchy. In the present case, the task rule can be assumed to be at the top of the hierarchical representation and the response to be at the bottom. Accordingly, a task switch resulted in a tendency to switch the responses. Again, this generalized switch must operate at the level of the hands and fingers, because $R$ was unaffected by switching in the bivalent response setup. In other words, a signal to switch hands (and hence tasks) resulted in a tendency to reverse the responding finger.

Because $T$ was quite high, the rate of task errors $(1-T)$ was not, yielding noisy estimates of $I$. We can only say that, at least in the bivalent setups, the effect of switching on $I$ was significant and tended in the same direction as $R$. This result cannot be explained by incremental reconfiguration, which would predict the reverse pattern. Specifically, the system is better reconfigured for the irrelevant task in switch trials than in nonswitch trials, which should result in more accurate executions of this irrelevant task. However, an uncontrollable application of a switch signal could have produced such an effect. In this case, the switch signal failed to reverse the task but succeeded in reversing the response.

\section{The Residual Switching Cost}

In the present experiments, we replicated the usual RT and PE residual task-switching costs. That is, switching cost was not abolished by preparation in the bivalent response setup but was abolished when the response setup was univalent (Brass et al., 2003; Meiran, 2000b; see Mayr, 2001). A surprising finding concerned the complete elimination of the $T$ switching cost by preparation even in the bivalent response setup.

We suggest that, from the present perspective, the RT residual cost does not necessarily reflect control failure. Instead, it reflects the fact that sufficient control was applied to reach the behavioral goal, which was to respond according to the instructed task. More control was simply unnecessary and possibly too costly to implement. In other words, we agree with Allport and his colleagues (Allport et al., 1994; Allport \& Wylie, 2000; Waszak et al., 2003) that the residual task-switching cost should be interpreted as a priming effect.

\section{Implications for Other Theories}

In this section, we discuss the implications of the present results for two additional theories. According to Dreisbach et al. (2002), participants do not use task-specific preparation (reconfiguration) but rather expectancy, which is a general form of preparation. Dreisbach et al. explain the overadditive interaction between switching and preparation time as reflecting the fact that participants expect task repetitions more than they expect task switches. This hypothesis could easily explain the overadditive interaction between switching and preparation time that we observed on PE and RT. However, the theory also makes the prediction that such overadditive interaction would also be observed in $R$, because expectancy should facilitate correct response selection. Although this is what we observed when univalent response setups were employed, there was no evidence of switch or CTI effects on $R$ in the 
bivalent response setup. We place more faith in the latter result because in the bivalent setup one can be certain only that task preparation was performed on a cognitive level rather than on the level of the responding hand or finger. We should add that Dreisbach et al.'s hypothesis predicts that the overadditive interaction on RT between switching and preparation time should be ubiquitous as long as switch expectancy is unchanged. This prediction is inconsistent with several findings in the literature which show that the presence of an overadditive interaction between switching and preparation time depends on reconfiguration demands or feasibility, and may even be completely absent in some cases (Mayr \& Kliegl, 2000; Meiran, 2000b; Meiran \& Marciano, 2002).

Another relevant theory is Logan and Bundesen's (2003). According to these authors, the cuing task-switching paradigm does not involve task switching. The effects of switching are explained by the switching or repetition of the task cue. The reduction in the switching effect with increased preparation time is expected because of the savings in task-cue processing time due to the opportunity to process it in advance. Our conceptual framework, presented in Figures 2 and 5, may be interpreted in terms of Logan and Bundesen's theory as reflecting the processing of the first presented element (the task cue) and that of the second presented element (the target stimulus), rather than task decision and response selection, respectively. However, there is one critical feature of the results that is somewhat inconsistent with that interpretation. Specifically, according to the new interpretation, $T$ reflects the probability of correctly classifying the task cue, and $R$ and $I$ represent the probability of correctly classifying the target stimulus. If this is accurate, there is every reason to expect $T$ to be greater than $R$ and $I$, because the former represents a two-choice classification for which more processing time was allowed (CTI + RT) whereas the latter represent four-choice classifications with less processing time (RT). In contrast to this expectation, we observed that $T$ was generally lower than $R$, especially in the bivalent response setup. Moreover, the fact that the $R$-versus- $T$ difference increased in the bivalent condition is to be expected when one assumes that task switching takes place, because bivalency influences task confusability. Another feature of the results that Logan and Bundesen's theory seems to have difficulty handling lies in the effects of switching and preparation on $R$, which we observed in the univalent response setups. We could come up with explanations based only on the assumption that switching takes place. In fact, we do not see how one could explain this bivalency disadvantage in $T$ and the effects on $R$ without the assumption of task switching. Of course, this could be due to our lack of imagination.

The present results are consistent with both versions of the priming hypothesis suggested by Allport and his colleagues and therefore cannot be used distinguish between them. According to the first hypothesis - that of task-set inertia (Allport et al., 1994) - the switching effect on $T$ could be due to the fact that the system's configuration remained in place after task execution. In switch trials, this means that the configuration occasionally failed to change on time, which resulted in the execution of the wrong task. Alternatively, because only four target stimuli were used, the results could also indicate the unintentional and counterproductive retrieval of the wrong task set by the target stimulus, due to stimulus-task binding (Allport \& Wylie, 2000; Waszak et al., 2003).

In conclusion, the present work concentrated on task errors. Our findings indicate that task switching increased the task error rate, whereas advance preparation eliminated this switching effect. The results allowed us to rule out a new variant of the priming hypothesis that could have explained RT and general PE. Because this hypothesis was ruled out, the present results provide further support of the hypothesis concerning priming, which is counteracted by advance reconfiguration.

\section{REFERENCES}

Allport, A., Styles, E. A., \& Hsieh, S. (1994). Shifting intentional set: Exploring the dynamic control of tasks. In C. Umiltà \& M. Moscovitch (Eds.), Attention and performance XV (pp. 421-452). Hillsdale, NJ: Erlbaum

Allport, A., \& Wylie, G. (2000). Task-switching, stimulus-response bindings, and negative priming. In S. Monsell \& J. Driver (Eds.), Control of cognitive processes: Attention and performance XVIII (pp. 35-70). Cambridge, MA: MIT Press.

Batchelder, W. H., \& Riefer, D. M. (1999). Theoretical and empirical review of multinomial process tree modeling. Psychonomic Bulletin \& Review, 6, 57-86.

BIEDERMAN, I. (1972). Human performance in contingent information processing tasks. Journal of Experimental Psychology, 93, 219-238.

Brass, M., Ruge, H., Meiran, N., Rubin, O., Koch, I., Zysset, S., ET AL. (2003). When the same response has different meanings: Recoding the response meaning in the lateral prefrontal cortex. NeuroImage, 20, 1026-1031.

Braver, T. S., Reynolds, J. R., \& Donaldson, D. I. (2003). Neural mechanisms of transient and sustained cognitive control during task switching. Neuron, 39, 713-726.

DE JONG, R. (1995). Strategical determinants of compatibility effects with task uncertainty. Acta Psychologica, 88, 187-207.

DE JONG, R. (2000). An intention-activation account of residual switch costs. In S. Monsell and J. Driver (Eds.), Control of cognitive processes: Attention and performance XVIII (pp. 357-376). Cambridge, MA: MIT Press.

Dreisbach, G., Haider, H., \& Kluwe, R. H. (2002). Preparatory processes in the task-switching paradigm: Evidence from the use of probability cues. Journal of Experimental Psychology: Learning, Memory, \& Cognition, 28, 468-483.

Duncan, J. (1977). Response selection rules in spatial choice reaction tasks. In S. Dornik (Ed.), Attention and performance VI (pp. 49-61). Hillsdale, NJ: Erlbaum.

Duncan, J. (1978). Response selection in spatial choice reaction: Further evidence against associative models. Quarterly Journal of Experimental Psychology, 30, 429-440.

FAGOT, C. (1994). Chronometric investigations of task switching. Unpublished doctoral dissertation, University of California, San Diego.

Gilbert, S. J., \& Shallice, T. (2002). Task switching: A PDP model. Cognitive Psychology, 44, 297-337.

Gotler, A., Meiran, N., \& Tzelgov, J. (2003). Nonintentional task set activation: Evidence from implicit task sequence learning. Psychonomic Bulletin \& Review, 10, 890-896.

Heuer, H., Schmidtke, V., \& Kleinsorge, T. (2001). Implicit learning 
of sequences of tasks. Journal of Experimental Psychology: Learning, Memory, \& Cognition, 27, 967-983.

Hillstrom, A. P., \& Logan, G. D. (1997). Process dissociation, cognitive architecture, and response time: Comments on Lindsay and Jacoby (1994). Journal of Experimental Psychology: Human Perception \& Performance, 23, 1561-1578.

Hommel, B. (1996). No prevalence of right-left over top-bottom spatial codes. Perception \& Psychophysics, 58, 102-110.

Hommel, B. (2000). The prepared reflex: Automaticity and control in stimulus-response translation. In S. Monsell \& J. Driver (Eds.), Control of cognitive processes: Attention and performance XVIII (pp. 247-274). Cambridge, MA: MIT Press.

Hübner, R., Futterer, T., \& Steinhauser, M. (2001). On attentional control as a source of residual shift costs: Evidence from twocomponent task shifts. Journal of Experimental Psychology: Learning, Memory, \& Cognition, 27, 640-653.

Jersild, A. T. (1927). Mental set and shift. Archives of Psychology (Whole Vol. 89)

Keele, S. W., \& Rafal, R. (2000). Deficits of task set in patients with left prefrontal cortex lesions. In S. Monsell \& J. Driver (Eds.), Control of cognitive processes: Attention and performance XVIII (pp. 627652). Cambridge, MA: MIT Press.

KLeINSORge, T., \& Heuer, H. (1999). Hierarchical switching in a multidimensional task space. Psychological Research, 62, 300-312.

KocH, I. (2001). Automatic and intentional activation of task sets. Journal of Experimental Psychology: Learning, Memory, \& Cognition, 27, 1474-1486.

Kofman, O., Meiran, N., Greenberg, E., Balas, M., \& Cohen, H. (in press). Enhanced performance on executive function tasks associated with examination stress. Cognition \& Emotion.

Kray, J., \& Lindenberger, U. (2000). Adult age differences in task switching. Psychology \& Aging, 15, 126-147.

LindSAY, D. S., \& JACOBY, L. L. (1994). Stroop process dissociations: The relationship between facilitation and interference. Journal of Experimental Psychology: Human Perception \& Performance, 20, 219-234.

Logan, G. D. (1994). On the ability to inhibit thought and action: A users' guide to the stop signal paradigm. In D. Dagenbach \& T. H. Carr (Eds.), Inhibitory processes in attention, memory, and language (pp. 189-239). San Diego: Academic Press.

LogAN, G. D., \& Bundesen, C. (2003). Clever homunculus: Is there an endogenous act of control in the explicit task cuing procedure? Journal of Experimental Psychology: Human Perception \& Performance, 29, 575-599.

Los, S. A. (1996). On the origin of mixing costs: Exploring information processing in pure and mixed blocks of trials. Acta Psychologica, 94, $145-188$

Los, S. A. (1999). Identifying stimuli of different perceptual categories in mixed blocks of trials: Evidence for cost in switching between computational processes. Journal of Experimental Psychology: Human Perception \& Performance, 25, 3-23.

Marble, J. G., \& Proctor, R. W. (2000). Mixing location-relevant and location-irrelevant choice-reaction tasks: Influences of location mapping on the Simon effect. Journal of Experimental Psychology: Human Perception \& Performance, 26, 1515-1533.

MAYr, U. (2001). Age differences in the selection of mental sets: The role of inhibition, stimulus ambiguity, and response-set overlap. Psychology \& Aging, 16, 96-109.

MaYr, U., \& KliEgL, R. (2000). Task-set switching and long-term memory retrieval. Journal of Experimental Psychology: Learning, Memory, \& Cognition, 26, 1124-1140.

Meiran, N. (1996). Reconfiguration of processing mode prior to task performance. Journal of Experimental Psychology: Learning, Memory, \& Cognition, 22, 1423-1442.

MeIRAn, N. (2000a). Modeling cognitive control in task-switching. Psychological Research, 63, 234-249.

Meiran, N. (2000b). Reconfiguration of stimulus task sets and response task sets during task switching. In S. Monsell \& J. Driver (Eds.), Control of cognitive processes: Attention and performance XVIII (pp. 377400). Cambridge, MA: MIT Press.
Meiran, N. (2005). Task rule congruency and Simon-like effects in switching between spatial tasks. Quarterly Journal of Experimental Psychology, 58A, 1023-1041.

Meiran, N., \& Chorev, Z. (2005). Phasic alertness and the residual task-switching cost. Experimental Psychology, 52, 109-124.

MeIran, N., ChOREv, Z., \& SAPIR, A. (2000). Component processes in task switching. Cognitive Psychology, 41, 211-253.

Meiran, N., Gotler, A., \& Perlman, A. (2001). Old age is associated with a pattern of relatively intact and relatively impaired task-set switching abilities. Journals of Gerontology: Psychological Sciences \& Social Sciences, 56B, P88-P102.

Meiran, N., Hommel, B., Bibi, U., \& LeV, I. (2002). Consciousness and control in task switching. Consciousness \& Cognition, 11, 10-33.

Meiran, N., Levine, J., Meiran, N., \& Henik, A. (2000). Task set switching in schizophrenia. Neuropsychology, 14, 471-482.

Meiran, N., \& Marciano, H. (2002). Limitations in advance task preparation: Switching the relevant stimulus dimension in speeded same-different comparisons. Memory \& Cognition, 30, 540-550.

Monsell, S., \& Driver, J. (EDS.) (2000). Control of cognitive processes: Attention and performance XVIII. Cambridge, MA: MIT Press.

MyUNG, I. J. (2003). Tutorial on maximum likelihood estimation. Journal of Mathematical Psychology, 47, 90-100.

Nicoletti, R., \& Umiltà, C. (1984). Right-left prevalence in spatial compatibility. Perception \& Psychophysics, 35, 333-343.

Nicoletti, R., \& Umiltà, C. (1985). Responding with hand and foot: The right/left prevalence in spatial compatibility is still present. Perception \& Psychophysics, 38, 211-216.

Riefer, D. M., \& BATChelder, W. H. (1988). Multinomial modeling and the measurement of cognitive processes. Psychological Review, 95, 318-339.

Rogers, R. D., \& Monsell, S. (1995). The cost of a predictable switch between simple cognitive tasks. Journal of Experimental Psychology: General, 124, 207-231.

Rosenbaum, D. A. (1980). Human movement initiation: Specification of arm, direction, and extent. Journal of Experimental Psychology: General, 109, 444-474.

Rubin, O., \& MeIRAN, N. (in press). On the origins of the task mixing cost in the cuing task switching paradigm. Journal of Experimental Psychology: Learning, Memory, \& Cognition.

Rubinstein, J. S., Meyer, D. E., \& Evans, J. E. (2001). Executive control of cognitive processes in task switching. Journal of Experimental Psychology: Human Perception \& Performance, 27, 763-797.

Ruge, H., Brass, M., Koch, I., Rubin, O., Meiran, N., \& von CraMON, D. Y. (2005). Advance preparation and stimulus-induced interference in cued task switching: Further insights from BOLD fMRI. Neuropsychologia, 43, 340-355.

Ruthruff, E., Remington, R. W., \& Johnson, J. C. (2001). Switching between simple cognitive tasks: The interaction of top-down and bottom-up factors. Journal of Experimental Psychology: Human Perception \& Performance, 27, 1404-1419.

SCHNEIDER, W. (1988). Micro Experimental Laboratory: An integrated system for IBM PC compatibles. Behavior Research Methods, Instruments, \& Computers, 20, 206-217.

SHAFFER, L. H. (1965). Choice reaction with variable S-R mapping. Journal of Experimental Psychology, 70, 284-288.

SimON, J. R., \& SMALL, A. M., JR. (1969). Processing auditory information: Interference from an irrelevant cue. Journal of Applied Psychology, 53, 433-435.

SoHn, M. H., \& ANDERSON, J. R. (2001). Task preparation and task repetition: Two-component model of task switching. Journal of Experimental Psychology: General, 130, 764-778.

SoHn, M. H., \& CARLSON, R. A. (2000). Effects of repetition and foreknowledge in task-set reconfiguration. Journal of Experimental Psychology: Learning, Memory, \& Cognition, 26, 1445-1460.

Stroop, J. R. (1935). Studies of interference in serial verbal reactions. Journal of Experimental Psychology, 18, 643-662.

SudeVAn, P., \& TAYLOR, D. A. (1987). The cuing and priming of cognitive operations. Journal of Experimental Psychology: Human Perception \& Performance, 13, 89-103.

VU, K.-P. L., \& ProctoR, R. W. (2001). Determinants of right-left and 
top-bottom prevalence for two-dimensional spatial compatibility. Journal of Experimental Psychology: Human Perception \& Performance, 27, 813-828.

Vu, K.-P. L., \& Proctor, R. W. (2002). The prevalence effect in twodimensional stimulus-response compatibility is a function of the relative salience of the dimensions. Perception \& Psychophysics, 64, 815-828.

Waszak, F., Hommel, B., \& Allport, A. (2003). Task-switching and long-term priming: Role of episodic stimulus-task bindings in taskshift costs. Cognitive Psychology, 46, 361-413.

Yeung, N., \& Monsell, S. (2003a). The effects of recent practice on task switching. Journal of Experimental Psychology: Human Perception \& Performance, 29, 919-936.
Yeung, N., \& Monsell, S. (2003b). Switching between tasks of unequal familiarity: The role of stimulus attribute and response-set selection. Journal of Experimental Psychology: Human Perception \& Performance, 29, 455-460.

Zelazo, P. D., \& Frye, D. (1997). Cognitive complexity and control: A theory of the development of deliberate reasoning and intentional action. In M. Stamenov (Ed.), Language structure, discourse, and the access to consciousness (pp. 113-153). Amsterdam: John Benjamins.

(Manuscript received September 9, 2003;

revision accepted for publication November 8,2004 .) 